\title{
»Freier Wille« und Schuld von Gewaltstraftätern aus Sicht der Hirnforschung und Neuropsychologie
}

\author{
Gerhard Roth, Monika Lück und Daniel Strüber
}

\section{Einleitung}

Kaum ein Thema erregt seit Jahren die Öffentlichkeit so sehr wie Gewalt, sei es in Form terroristischer Akte, ausländerfeindlicher brutaler Gewalt oder Sexualmorde an Kindern. Es erhebt sich dann mehr oder weniger automatisch die Frage: »Warum können Menschen so etwas tun?» Parallel hierzu wird - insbesondere auf Grund neuer Untersuchungen von Neurobiologen und Psychologen - in philosophischen, juristischen und soziologischen Fachkreisen und in der akademisch gebildeten Öffentlichkeit die Frage der Willensfreiheit und der Verantwortlichkeit für das eigene Tun intensiv und, wie nicht anders zu erwarten, kontrovers diskutiert. Das wachsende Interesse der Juristen ist verständlich, gehört die Debatte um die Willensfreiheit doch zu den großen Themen des deutschen bzw. kontinentaleuropäischen Strafrechts. Hier hat es neben Befürwortern eines auf Immanuel Kant zurück gehenden »starken« Begriffs von Willensfreiheit auch immer Willensfreiheits-Skeptiker wie Franz von Liszt und Gustav Radbruch oder Agnostiker wie Claus Roxin gegeben - um nur wenige Namen zu nennen. Der »starke« Begriff von Willensfreiheit lässt sich im Prinzip des Alternativismus, des Anders-HandelnKönnens, zusammenfassen: Wenn ein Täter wusste, was er tut, und wenn er im normalen Leben imstande ist, seine Handlungen bewusst zu planen und zu lenken, dann besitzt er auch die Möglichkeit, allein kraft seines (moralischen) Willens seine Taten so zu lenken, dass sie gesetzestreu sind, und er damit den Motiven, die ihn zur Tat treiben, zu widerstehen. Schuldig wird er dadurch, dass er von dieser Möglichkeit und seiner Einsicht in Recht und Unrecht willentlich nicht Gebrauch gemacht und Unrecht begangen hat. Schuld gibt es nur, wenn es einen freien Willen in diesem starken, alternativistischen Sinne gibt. Ein durchgehender Determinismus, gleichgültig ob sozialer, psychologischer oder neurobiologischer Art, ist danach mit Willensfreiheit und Schuld unvereinbar-inkompatibel (vgl. hierzu Pauen, 2004).

Während weder die Diskussion um die Willensfreiheit selbst noch die philosophischen und juristischen Argumente für oder wider neu sind (dies gilt auch für den »schwachen« oder kompatibilistischen Begriff von Willensfreiheit, der nicht auf dem Prinzip des Alternativismus aufbaut), gibt es doch zwei neue und wesentliche Gesichtspunkte, nämlich zum einen neue experimentell-empirische Einsichten der Neurowissenschaften und der Psychologie bzw. Psychiatrie in das Entstehen von Charakter und Persönlichkeit, von Handlungsmotiven und deren Realisierung bzw. Nichtrealisierung und in die komplizierte Beziehung bewusster Handlungskontrolle und unbewussten Antrieben (s. hierzu Roth, 2003). Entsprechend lassen sich Zweifel an dem oben genannten »starken « Begriff von Willensfreiheit nicht mehr als rein philosophische Spekulationen abtun. Zum zweiten gibt es zahlreiche retrospektive und inzwischen auch prospektive Studien zu den Merkmalen, die chronische Gewaltstraftäter charakterisieren, und diese Studien kommen zu erstaunlich übereinstimmenden Ergebnissen (Überblick in Lück, Strüber \& Roth, 2005).

Zusammengefasst zeigen diese Studien, dass Gewaltverhalten einerseits nicht aufgrund eines einzigen Faktors wie »die Gene« oder »die Umwelt « erklärt werden kann, vielmehr erhöht sich die Wahrscheinlichkeit, gewalttätig zu werden, durch das Zusammenspiel verschiedener sowohl individueller biologisch-psychologischer als auch sozialer Risikofaktoren. Zum anderen aber werden diese Faktoren alle entweder schon vor der Geburt oder in früher Kindheit wirksam - zu einer Zeit also, in der die betroffenen Personen noch gar nicht schuldfähig sind. Hierzu gehören (1) Geschlecht, (2) Alter, (3) genetisch oder entwicklungsbedingte hirnanatomische und -physiologische Störungen, (4) eine gestörte frühkindliche Bindungserfahrung, (5) traumatische frühkindliche Ereignisse, insbesondere Vernachlässigung, körperliche Misshandlung und sexueller Missbrauch, und (6) ungünstige familiäre soziale Bedingungen wie Armut, elterliche Konflikte, Gewaltbereitschaft der näheren sozialen Umgebung. Im Folgenden sollen einige dieser Faktoren kurz besprochen werden, ehe auf die Frage nach der Schuldfähigkeit der Gewalttäter eingegangen wird.

\section{Die Bedeutung von Geschlecht und Alter}

Es sind überwiegend männliche Jugendliche und Erwachsene, die körperliche Gewaltdelikte begehen. Nach Angaben des Statistischen Bundesamts für die Bundesrepublik Deutschland wurden im Jahre 2004 wegen Gewaltdelikten (Vergewaltigung, sexueller Missbrauch, Körperverletzung, gefährliche und schwere Körperverletzung sowie Mord und Totschlag) 44.029 über 21-Jährige verurteilt (Daten nur für die alten Bundesländer vorhanden, Statistisches Bundesamt, 2006). Davon waren 41.076 Männer und 2.953 Frauen. Bei schweren Gewaltdelikten ist der Unterschied besonders dra- matisch: Wegen Mordes und Totschlags wurden im Jahre 2004521 Männer und 37 Frauen verurteilt, wegen gefährlicher und schwerer Körperverletzung 11.453 Männer und 907 Frauen, wegen Vergewaltigung 673 Männer und 1 Frau und wegen sexuellen Missbrauchs von Kindern 2049 Männer und 23 Frauen. Ähnliche Größenordnungen finden sich auch in anderen westeuropäischen Ländern sowie den USA.

Ganz allgemein lässt sich feststellen, dass das männliche Geschlecht umso deutlicher dominiert, je schwerer die Gewalttat ist. Dies heißt nicht, dass Mädchen bzw. Frauen »von Natur aus« weniger aggressiv sind, sondern nur, dass Jungen/Männer eher zu direkter, nach außen gerichteter körperlicher Gewalt neigen, Mädchen / Frauen hingegen eher zu indirekter Gewalt, d. h. verdeckter Aggression mittels sozialer Manipulation (Björkqvist et al., 1992), oder zu autoaggressiven Handlungen. Die vorliegenden Studien zeigen, dass die meisten Gewaltkarrieren um das 12. Lebensjahr beginnen, sich im Alter zwischen 13 und 14 Jahren verdoppeln, weiter zunehmen bis zu einem Höhepunkt von 16 bis 17 Jahren, dann im Alter von 18 Jahren um die Hälfte abnehmen und sich kontinuierlich verringern bis zum 27. Lebensjahr. Demgegenüber steht eine kleine Gruppe, ca. $5 \%$ der männlichen Jugendlichen, die sehr früh aggressives bzw. antisoziales Verhalten zeigen und darin nicht nachlassen (Moffitt \& Caspi, 2001). Neuere Untersuchungen zeigen allerdings, dass neben diesen beiden klar definierten Gruppen von Gewalttätern auch abweichende Entwicklungsverläufe möglich sind (Loeber et al., 2005; Moffitt et al., 2002).

\section{Neurobiologische Auffälligkeiten bei Gewaltstraftätern}

Hinsichtlich der neurobiologischen Korrelate gewalttätigen Verhaltens ist mindestens zwischen zwei Formen von Aggression zu unterscheiden, nämlich impulsiver und instrumenteller Aggression. Impulsive Aggression am häufigsten $\mathrm{zu}$ finden und tritt als Reaktion auf erlebte Bedrohungen, Frustrationen oder Provokationen auf und ist durch eine starke emotionale Beteiligung (Ärger, Wutausbrüche) gekennzeichnet. Instrumentelle Aggression hingegen ist offenbar wesentlich seltener; sie ist geplant und auf das Erreichen eines angestrebten Zieles (Besitz, Status) hin ausgerichtet und wird häufig mit geringem emotionalem Aufwand ausgeführt. Möglicherweise gibt es noch 
eine dritte Form lustvoller Aggression, die ausgeführt wird, um einen besonderen Kitzel oder Rausch $\mathrm{zu}$ erleben (eine besondere Form von »sensation seeking «) oder eine innere Leere auszufüllen. Obwohl diese unterschiedlichen Aggressionsformen alle mit schweren Gewaltstraftaten in Beziehung stehen können, sind die zugrunde liegenden Hirnmechanismen bzw. Hirnpathologien durchaus unterschiedlich, wie im Folgenden dargestellt. Dies wiederum hat Konsequenzen für die Therapierbarkeit.

Gewaltverbrecher mit erhöhter impulsiver Aggressivität zeigen gegenüber der Normalpopulation signifikant häufiger bestimmte strukturelle und funktionale Hirndefizite im Bereich des Stirnhirns (präfrontaler, orbitofrontaler, anteriorer cingulärer und insulärer Cortex) und des Schläfenlappens (Temporalcortex) sowie in limbischen Regionen (vornehmlich Amygdala und basales Vorderhirn), die alle mit der Entstehung und der Kontrolle affektiver und emotionaler Zustände zu tun haben (Bogerts, 2004; Bufkin \& Luttrell, 2005; Davidson et al., 2000; Raine et al., 1997; 2000; für eine Übersicht s. Roth, 2003; Roth \& Dicke, 2005). Eine Verminderung der Aktivität des Frontalhirns durch Läsionen oder Fehlentwicklungen führt zu einer erhöhten Risikobereitschaft, einer gesteigerten Impulsivität und "unmoralisch«-kriminellem Verhalten (Bechara et al., 1997; Brower \& Price, 2001).

Eine besondere Rolle spielt hierbei der orbitofrontale, d.h. über den Augenhöhlen (Orbita) liegende Cortex (OFC). Personen mit Schädigungen des OFC in frühester Jugend zeigten schwer antisoziales Verhalten auch beim Aufwachsen in normaler Umgebung, sie sind unerziehbar und unbelehrbar (Anderson et al., 1999). Allgemein wird davon ausgegangen, dass die genannten Bereiche des Stirnhirns eine hemmende beziehungsweise zügelnde Wirkung auf subcorticale (d.h. außerhalb des Cortex liegende) limbische Zentren ausüben, insbesondere auf die Amygdala, das basale Vorderhirn, den Hypothalamus und das zentrale Höhlengrau, von denen negative Emotionen und aggressive Impulse ausgehen können. Sind die hemmenden Nervenbahnen vom OFC auf diese subcorticalen limbischen Zentren beeinträchtigt, so fällt dieser zügelnde Einfluss fort und es dominiert impulsives, gewalttätiges Verhalten. In diesem Zusammenhang ist es wichtig, dass der OFC derjenige Hirnteil ist, der am spätesten ausreift, nämlich erst mit 18 bis 20 Jahren, und nicht zufällig kommen die meisten Personen dann zu einiger Vernunft und Impulskontrolle.

Bei hochaggressiven und gewalttätigen Personen liegen außer diesen anatomischen Hirndefiziten deutliche physiologische Veränderungen vor, die vor allem die so genannten Neurotransmitter bzw. -modulatoren Serotonin und Dopamin betreffen. Serotonin hat generell einen beruhigenden und angstmindernden Effekt und spielt eine wichtige Rolle bei der Impulskontrolle; ein Serotoninmangel begünstigt das Zustandekommen impulsiv-gewalttätigen Verhaltens (Lee \& Coccaro, 2001). Dabei ist allerdings noch unklar, inwieweit ein niedriger Serotoninspiegel und Aggression direkt zusammenhängen oder über eine verstärkte negative Emotionalität (Furcht bzw. Ängstlichkeit, Bedrohtheitsgefühl, niedrige Frustrationstoleranz) als »reaktive Aggression « vermittelt wird (»ich fühlte mich angegriffen, da musste ich mich doch wehren!«).

Die genetische Ausstattung des serotonergen Systems kann interindividuell erheblich variieren. Entsprechend werden unterschiedliche Genvarianten (so genannte Polymorphismen) beschrieben, die in Abhängigkeit von sozialen Umweltfaktoren eine Prädisposition für antisoziales und gewalttätiges Verhalten darstellen können (Lesch \& Merschdorf, 2000). Für erhöhte impulsive Aggressivität bei Normalprobanden scheint insbesondere ein Polymorphismus des so genannten TryptophanHydroxylase-Gens, das für die Serotonin-Synthese notwendig ist, eine wichtige Rolle $\mathrm{zu}$ spielen (Hennig et al., 2005; Manuck et al., 1999; New et al., 1998). Bei männlichen Gewalttätern besteht ein signifikant negativer Zusammenhang zwischen Gewalttätigkeit oder -bereitschaft und SerotoninSpiegel bzw. dessen Abbauprodukt 5-Hydroxyindolessigsäure (5-HIAA). Bei diesen Personen sind vor allem der 5-HT $1 \mathrm{~A}^{-}$und $5-\mathrm{HT}_{1 \mathrm{~B}}$-Rezeptor betroffen. Diese haben eventuell unterschiedliche Funktionen bei kognitiver und motorischer Impulsivität.

Aktuelle Befunde gibt es inzwischen auch zum so genannten 5-HT-Transporter, der Serotonin aus dem synaptischen Spalt entfernt und somit seine Wirksamkeit als Neurotransmitter beendet. Diese Befunde zeigen einen deutlichen Zusammenhang zwischen einem Gen-Polymorphismus, der die Funktion des 5-HT-Transporters verändert, und gewalttätigem Verhalten (Retz et al., 2004). Allerdings wurde in dieser Studie nicht zwischen impulsiven und instrumentellen Gewalttätern unterschieden. Mithilfe der Positronen-Emissions-Tomographie (PET) konnte eine negative Korrelation zwischen dem Bindungspotenzial des 5- $\mathrm{HT}_{1 \mathrm{~A}}$-Rezeptors in verschiedenen Gehirnbereichen und der mittels Fragebogen erhobenen Aggression bei Normalprobanden aufgezeigt werden (Parsey et al., 2002).

Für die vorliegende Frage der Kausalität von Gewalttäterschaft ist besonders die Tatsache bedeutsam, dass Defizite im Serotonin-Haushalt nicht nur genetisch bedingt, sondern auch die Folge negativer Umwelteinflüsse sein können. Vorgeburtliche Störungen des Serotonin-Haushalts, z. B. aufgrund eines chronischen mütterlichen Stresses oder Drogenkonsums während der Schwangerschaft, können schwere Entwicklungsstörungen hervorrufen. Der spätere Serotoninspiegel wird ebenso beeinflusst von schädlichen frühkindlichen Umwelteinflüssen wie Vernachlässigung, Gewalt und Missbrauch (Caspi et al., 2002; Huang et al., 2004).
Insgesamt gilt, dass das Zusammentreffen bestimmter genetisch, entwicklungsmäßig oder umweltbedingter kognitiver und emotionaler Risikofaktoren in der Kindheit für die Prognose der weiteren Entwicklung von Störungen des Sozialverhaltens, die sich in extremem Gewaltverhalten ausdrücken können, besonders ungünstig ist. Dazu zählen u.a. das Aufmerksamkeits-Defizit-Hyperaktivitäts-Syndrom (ADHS), mangelnde Impulskontrolle, Schwierigkeiten der Wahrnehmung und Interpretation sozialer Informationen, fehlende Empathiefähigkeit, Bindungsstörungen und klinisch auffälliges oppositionelles Trotzverhalten, bzw. Störungen des Sozialverhaltens (vgl. u. a. Carlo et al., 1999; Dodge et al., 2003; Eisenberg \& Morris, 2002; Lahey et al., 2000; Moffitt \& Caspi, 2001). Die Entstehung solcher Entwicklungsstörungen liegt in einem Zusammenspiel von individuellen, dem Kind eigenen biologisch-psychologischen Faktoren und der sozialen Umwelt. Vor allem die frühe Interaktion des Kindes mit seinen Bezugspersonen spielt hierbei eine große Rolle. Extrem widrige Entwicklungsumstände wie körperliche und sexuelle Misshandlung, Verwahrlosung, Gewalterfahrungen im näheren Umfeld und konfliktbeladene Eltern-Beziehungen stellen starke Risikofaktoren für massive Störungen der emotionalen und kognitiven Entwicklung des Kindes dar. Sie können im weiteren Lebenslauf zu schweren psychischen Störungen und insbesondere bei Männern zu einer erhöhten Neigung zu Gewalt führen.

In letzter Zeit wurden erste neurophysiologische Ergebnisse veröffentlicht, die einen solchen Zusammenhang zwischen strukturellen oder physiologischen Maßen und Verhaltensauffälligkeiten in der Kindheit darstellen, und zwar häufig schon in der frühen Kindheit oder sogar vor der Geburt. Wichtige Schritte der neuronalen Entwicklung finden schon in der Schwangerschaft statt, aber auch nach der Geburt entwickelt sich das Gehirn weiter, in bestimmten Regionen bis nach der Pubertät. Die Plastizität des kindlichen Gehirns dient unter anderem dazu, dass sich ein Individuum an seine soziale Umwelt anpasst. Wie weiter oben schon erwähnt, wird die Hirnentwicklung dabei neben genetischen Faktoren durch Erfahrungen bestimmt (für eine ausführlichere Darstellung vgl. Cicchetti, 2002) und wird dadurch empfänglich für negative Umwelteinflüsse.

Ein Beispiel für den engen Zusammenhang zwischen psychosozialen Risikofaktoren und (Neuro) Physiologie findet sich in einer Längsschnittstudie (Mannheimer Risikokinderstudie), die bei Jungen mit einer erhöhten Tendenz zu allgemein externalen, d. h. nach außen gerichteten Verhaltensstörungen ein erhöhtes Maß an 5a-Dihydro-Testosteron fand (Maras et al., 2003). Ebenso zeigte sich bei Kindern, deren Interaktion mit ihrer Bezugsperson durch eine unsichere Bindung charakterisiert war oder die in ihrer frühen Kindheit misshandelt wurden, ein erhöhter Cortisol Wert in Stress-Situationen (Burgess et al., 2003; Spangler, Grossmann \& Schieche, 2002). 
Während - wie oben dargestellt - der Zusammenhang zwischen einem niedrigen Serotoninspiegel und erhöhter Gewaltbereitschaft bei Erwachsenen (überwiegend bei Männern) deutlich ist, fehlen entsprechende eindeutige Befunde bisher bei Kindern. In letzter Zeit wurden und werden jedoch verstärkt Versuche unternommen, solche Zusammenhänge aufzudecken (Clarke et al., 1999; van Goozen et al., 1999). Der Mannheimer Risikokinderstudie entstammen ebenfalls Daten zum Zusammenhang zwischen externalen Verhaltensauffälligkeiten und erhöhter Neigung zu körperlicher Gewalt mit der Serotoninkonzentration im Blutplasma (Maras et al., 2006). Gefunden wurde ein negativer Zusammenhang zwischen Serotonin und den Werten der Problemskala »Aggression« und "Externale Probleme« der Child-BehaviorCheck-List (Achenbach, 1991). So wiesen Kinder mit erhöhten Werten auf den entsprechenden Skalen einen niedrigeren Serotoninspiegel auf. Aggressivität könnte in diesem Zusammenhang mindestens zwei Ursachen haben, die beide mit einem niedrigen Serotoninspiegel verbunden sind, nämlich erstens ein primäres affektiv-emotionales Defizit, das zu einer generellen Fehleinschätzung von Umweltereignissen führt, und zweitens ein Gefühl der Bedrohtheit und Unsicherheit als Folge frühkindlicher sozialer Isolation (vgl. Heinz, 2000).

\section{Psychopathie - Neuronale Korrelate instrumenteller Gewalt?}

Die bisher dargestellten Zusammenhänge bezogen sich auf die Ursachen "impulsiven « Gewaltverhaltens, wie es für den größten Teil von Gewaltstraftätern charakteristisch ist. Hier finden sich signifikante Zusammenhänge zwischen anatomischen und physiologischen Hirndefiziten, insbesondere was Frontalhirnschäden und Störungen des Serotoninhaushalts betrifft. Es gibt aber eine kleine Gruppe von besonders gefährlichen Gewalttätern und Mördern, die ihre Taten sorgsam planen und kaltblütig, »mitleidlos « und ohne Schuldgefühle ausführen. Sie werden meist als »Psychopathen « oder "Soziopathen « bezeichnet und erregen die besondere Abscheu der Bevölkerung, insbesondere wenn sie vor Gericht keine Reue zeigen (dies zieht meist die Feststellung der »besonderen Schwere des Verbrechens« nach sich).

Während bei den »reaktiven", d. h. aus einem starken Gefühl des Bedrohtseins heraus handelnden Gewaltstraftätern eine deutliche Beeinträchtigung der für die Affektregulation verantwortlichen Stirnhirnbereiche vorliegt, weist demgegenüber der kaltblütig planende Gewalttäter eine weitgehend intakte Funktion der Stirnhirnregionen auf einschließlich des oberen Stirnhirns (des so genannten dorsolateralen präfrontalen Cortex, PFC), der Sitz von Intelligenz, Verstand und Handlungsplanung ist. Dies ist nicht überraschend, denn langfristige Planung einer Handlung erfordert komplexe Entscheidungsprozesse, die es erlauben, zweckgerichtet zu agieren und die Konsequenzen der erforderlichen Teilhandlungen richtig einzuschätzen. Ein wichtiges zusätzliches Motiv für den planenden Gewalttäter besteht darin, nach Möglichkeit nicht »erwischt « zu werden (worüber der impulsive Gewalttäter nicht nachdenkt). Entsprechend könnte es sein, dass sich bisher unentdeckte Straftäter von gefassten und verurteilten Straftätern hinsichtlich ihrer kognitiv-affektiven Hirnfunktionen unterscheiden.

Dieser Frage wurde kürzlich in einer Untersuchungsreihe von Adrian Raines Arbeitsgruppe nachgegangen. Verglichen wurden zwei Gruppen so genannter krimineller Psychopathen, die zwar beide schwere Gewalttaten begangen hatten, aber nur zum Teil entdeckt und für ihre Straftaten verurteilt wurden. Die bisher unentdeckt Gebliebenen bezeichnete Raine als »erfolgreiche Psychopathen « (successful psychopaths), während die verurteilten Gewalttäter als »erfolglose Psychopathen « (unsuccessful psychopaths) eingestuft wurden. Ein hirnanatomischer Vergleich dieser beiden Gruppen mit einer Kontrollgruppe zeigte nur für die erfolglosen, d. h. »erwischten « und verurteilten Gewaltstraftäter eine signifikante Volumenreduktion der grauen Substanz im präfrontalen Cortex. Hingegen unterschieden sich die erfolgreichen Psychopathen diesbezüglich nicht von Normalpersonen als Kontrolle (Yang et al., 2005).

Dieser Befund könnte dafür sprechen, dass Gewalttätigkeit nicht per se an präfrontale Hirnfunktionsdefizite gebunden ist, wie es bisherige Untersuchungen an verurteilten Straftätern vermuten ließen. Zumindest für Personen mit einer psychopathischen Persönlichkeit scheint es eine Untergruppe von Kriminellen zu geben, die regelmäßig schwere Gewalttaten begehen, obwohl sie über ein weitgehend intaktes Frontalhirn verfügen. Zudem sind sie aufgrund der darauf beruhenden Funktionsfähigkeit kognitiver Prozesse sogar »erfolgreich « und bleiben unentdeckt. Demnach stehen gestörte Frontalhirnfunktionen nicht mit dem Gewaltpotenzial als solchem in Zusammenhang, sondern erhöhen lediglich das Risiko der Täter, entdeckt zu werden. Infolge affektiv-kognitiver Defizite gehen sie impulsiv und planlos vor und können Signale nicht wahrnehmen, die Gefahr und mögliche Gefangennahme anzeigen.

Der präfrontale Cortex ist nur eines von vielen Zentren innerhalb eines komplexen Netzwerkes zur Regulation affektiv-emotionaler Zustände einschließlich aggressiven und antisozialen Verhaltens. Für eine Beteiligung limbischer Strukturen außerhalb der Großhirnrinde im engeren Sinne sprechen weitere Untersuchungen Raines an der selben Stichprobe krimineller Psychopathen. Dabei zeigten sich deutliche Größenunterschiede zwischen dem rechten und dem linken Hippocampus (Raine et al., 2004) Derartige Unterschiede in der Hippocampusgröße beider Gehirnhälften werden mit früh in der Hirnentwicklung aufgetretenen Entwicklungsstörungen in Verbindung gebracht, während eine beidseitige Verkleinerung des Hippocampus vorwiegend als Folge späterer Umwelteinflüsse angesehen werden, insbesondere von chronischem Stress (vgl. Bremner et al., 2003). Der Hippocampus ist der wichtigste Organisator des deklarativen, d. h. bewusstseinsfähigen und berichtbaren Langzeitgedächtnisses und damit auch einer der »Kontrolleure « unserer Gedanken und Wünsche. Kürzlich konnte nachgewiesen werden, dass der Hippocampus beim Prozess der »Verdrängung « im Freud'schen Sinne eine wichtige Rolle spielt (Anderson et al., 2004).

Insofern lässt sich der Befund einer strukturellen Hippocampus-Asymmetrie bei Psychopathen als Anzeichen einer Störung der frühen neurokognitiven Entwicklung interpretieren, die zu einer Beeinträchtigung der hippocampal-präfrontalen Regulationsschleife führt. Insbesondere in Kombination mit einer gestörten Verbindungen zwischen Hippocampus und Amygdala kann es zu einer falschen Verarbeitung emotionaler Informationen und zu unangemessenen emotionalen Äußerungen und Reaktionen kommen. Beides ist als klinisches Symptom bei Psychopathen beschrieben worden, die sich in Sicherheitsverwahrung befinden.

In der Tat zeigen die Ergebnisse von Raine eine strukturelle Hippocampus-Asymmetrie nur bei den entdeckten und damit erfolglosen Psychopathen. Die unentdeckt gebliebenen und damit erfolgreichen Psychopathen unterschieden sich wiederum nicht von der Kontrollgruppe (Raine et al., 2004). Zudem konnte in einer weiteren Untersuchung gezeigt werden, dass sich verminderte kardiovaskuläre Reaktionen auf emotionalen Stress (Herzschlag, Blutdruck) nur bei den erfolglosen Psychopathen zeigten. Erfolgreiche Psychopathen hingegen hatten sogar eine gegenüber normalen Kontrollpersonen und erfolglosen Psychopathen erhöhte Stressreagibilität und zeigten bessere Leistungen in neuropsychologischen Tests zur Überprüfung der Frontalhirnfunktion (Ishikawa et al., 2001).

Die differenzierte neurobiologische Untersuchung erfolgreicher Psychopathen ist ein neues und schwieriges Forschungsgebiet, da man bisher unentdeckte, also auf »freiem Fuß « befindliche, Psychopathen ausfindig machen und dazu bringen muss, über ihre Straftaten zuverlässig Auskunft zu geben. Dies erklärt, warum bisherige Untersuchungen ausschließlich an untergebrachten bzw. inhaftierten psychopathischen Gewalttätern durchgeführt wurden. Raines Ergebnisse zeigen, dass Täter mit der selben Diagnose der Persönlichkeitsstörung »Psychopathie« und vergleichbarem Gewaltpotenzial sehr unterschiedliche psychophysiologische und neurobiologische Korrelate aufweisen. Erfolgreiche Psychopathen unterscheiden sich in den von Raine untersuchten Funktionen nicht von gesunden Kontrollpersonen.

Sollte sich dieser Befund bestätigen, so hätten wir mit dem Typ des »erfolgreichen Psychopathen « ein vollkommen anderes Verursachungs- 
muster gewalttätigen Verhaltens vor uns, nämlich eine Person, die trotz voll funktionsfähiger Gehirnfunktionen und intakter Impulskontrollmechanismen Straftaten bewusst zum eigenen Vorteil begeht. Ein solcher Schluss ist sicherlich verfrüht. Beispielsweise ist die wichtige Funktion der Amygdala und des mesolimbischen Systems als zerebrales »Belohnungs- und Belohnungserwartungssystem « bisher noch nicht genügend berücksichtigt worden. Gerade Fehlfunktionen dieser Strukturen werden von einigen Forschern vornehmlich für psychopathisches Verhalten verantwortlich gemacht (Blair, 2005).

\section{Abschlussbetrachtung}

Die große Mehrzahl der bisher untersuchten Vielfach-Gewalttäter weist neben besonderen genetischen Merkmalen, die sich auf einen veränderten Serotonin-Stoffwechsel beziehen, deutliche neuroanatomische, neurophysiologische oder neuropsychologische Defizite auf, die in aller Regel bereits in der Kindheit und Jugend sichtbar werden. Wichtig zu beachten ist aber, dass diese Defizite allein eine Person aber offensichtlich nicht zu einer späteren Gewalttäterschaft prädestinieren, sondern - von sehr schweren hirnanatomischen und physiologischen Beeinträchtigung abgesehen - lediglich eine erhöhte Verletzbarkeit (Vulnerabilität) darstellen. In Kombination mit negativen psychosozialen Faktoren wie defizitäre Bindungserfahrungen (z. B. Vernachlässigung durch die Bezugsperson), körperlicher Misshandlung, sexuellem Missbrauch und Gewalterfahrungen in der nahen sozialen Umgebung führen sie aber mit hoher Wahrscheinlichkeit zu chronischer Gewalttäterschaft. Einzeln genommen können die genannten Risikofaktoren durchaus durch kompensatorische Hirnentwicklungen oder günstige psycho-soziale Umstände in ihrer Auswirkung gehemmt oder gemildert werden. Entsprechend gibt es Patienten mit neuroanatomischen Defiziten im Bereich des Stirnhirns und des limbischen Systems, die keine Gewalttäter sind. In diesen Fällen wurden die Funktionen von anderen Hirnteilen übernommen. Zur Zeit ist ganz unklar, warum bei den einen Personen kompensatorische Hirnentwicklungsprozesse stattfinden, bei den Gewaltstraftätern aber nicht.

In jedem Fall aber handelt es sich bei den Gewaltstraftätern um Faktoren, die meist ihre Wirkung bereits vorgeburtlich, in der Kindheit und frühen Jugend entfalten und nicht der Willensbildung des Straftäters unterliegen. Niemand kann für seine Gene, seine Gehirnentwicklung, für traumatisierende Erlebnisse im Kindesalter und ein negatives soziales Umfeld verantwortlich gemacht werden und damit auch nicht für eine daraus resultierende Neigung zu körperlicher Gewalt.

In diesem Fall ist es nicht zu rechtfertigen, dass den Tätern ein abstraktes »Anders-Handeln-Können « unterstellt wird - ganz abgesehen von der hier nicht diskutierten Frage, ob ein solches An-
ders-Handeln-Können auch für »Normalpersonen« überhaupt zutrifft. Sofern sich die hier genannten Erkenntnisse weiter erhärten, muss die Schuldfähigkeit chronischer Gewalttäter im Sinne eines alternativistischen "starken « Begriffs von Willensfreiheit und Schuld grundsätzlich überdacht werden. Besonders problematisch wird dies bei den psychopathischen Gewalttätern, die oft mit großer Umsicht und Gefühlskälte vorgehen, was vor Gericht in aller Regel strafverschärfend wirkt. Zweifellos spielt bei solchen »spektakulären« Fällen besonders grausamer und planvollen Verbrechens die öffentliche Empörung und der Ruf nach Sühne eine erkenntnis-hindernde Rolle.

Es ist zu erwarten, dass sich in den kommenden Jahren aufgrund einer intensiven Kooperation der beteiligten Disziplinen die Möglichkeiten hinsichtlich einer Frühdiagnose der genannten Risikofaktoren in den kommenden Jahren stark verbessern. Es ist in diesem Zusammenhang dringend notwendig, normale »jugendliche Raufbolde« von Kindern und Jugendlichen mit einer konstitutionellen Neigung zu körperlicher Gewalt zu unterscheiden - eine Unterscheidung, die heute noch nicht verlässlich möglich ist. Eine verfeinerte Diagnostik würde es außerdem ermöglichen, die Therapiemöglichkeiten, beziehungsweise im Idealfall die Präventionsansätze, genauer auf die individuelle Entwicklungsgeschichte zuzuschneiden und mögliche Defizite besser aufzugreifen.

Bedauerlicherweise wird in der Öffentlichkeit der Ruf immer lauter, verhaltensauffällige Jungen und junge Männer präventiv »wegzusperren «. Dies wäre jedoch in hohem Maße unethisch, denn rein statistisch gesehen wird die Mehrheit dieser Personen nicht zu chronischen Gewalttätern. Es mag sich hier um eine »Selbstreparatur« des Gehirns oder um kompensatorische psychosoziale Bedingungen handeln. Es muss vielmehr mit aller Kraft erreicht werden, dass junge Menschen, die zu körperlicher Gewalt neigen, eine geeignete Therapie erhalten, und zwar unabhängig davon, ob eine Person nur vorübergehend erhöht gewaltbereit ist (und sich diese Neigung später »auswächst«), oder ob er tatsächlich in hohem Maße gefährdet ist dies ist eben bisher nicht zu entscheiden.

Für das Strafrecht kann all dies nur bedeuten, zumindest bei den chronischen Gewalttätern auf das Prinzip der Strafe als Sühne, das aus dem Begriff der moralischen Schuld erwächst, zu verzichten und sich auf das Prinzip der Abschreckung, der Therapie und des Schutzes der Gesellschaft vor nachweislich nicht therapierbaren Straftätern zu beschränken, wie dies bereits von zahlreichen führenden Strafrechtstheoretikern konzipiert wurde.

\section{Kontakt}

Prof. Gerhard Roth, Institut für Hirnforschung, Universität Bremen, Hanse-Wissenschaftskolleg Delmenhorst; Monika Lück und Daniel Strüber, Hanse-Wissenschaftskolleg Delmenhorst.

\section{Literatur}

Achenbach, T. M. (1991). Manual for the Child Behavior Checklist/4-18 and 1991 Profile. Burlington: University of Vermont, Department of Psychiatry.

Anderson, M. C., Ochsner, K. N., Kuhl, B., Cooper, J. Robertson, E., Gabrieli, S. W., Glover, G. H. und Gabrieli, J. D. E. (2004): Neural systems underlying the suppression of unwanted memory. Science 303: 232235.

Anderson, S. W., Bechara, A., Damasio, H., Tranel, D. \& Damasio, A. R. (1999). Impairment of social and moral behavior related to early damage in human prefrontal cortex. Nature Neuroscience, 2(11), 10321037.

Bechara, A., Damasio, H., Tranel, D. \& Damasio, A. R. (1997). Deciding advantageously before knowing the advantageous strategy. Science, 275(5304), 1293-1295.

Björkqvist, K., Lagerspetz, K. M. J. \& Kaukiainen, A. (1992). Do girls manipulate and boys fight? Developmental trends regarding direct and indirect aggression. Aggressive Behavior, 18, 117-127.

Blair, R. J. R. (2005). Applying a cognitive neuroscience perspective to the disorder of psychopathy. Development and Psychopathology, 17, 865-891.

Bogerts, B. (2004). Gewalttaten aus der Sicht der Hirnforschung. Forensische Psychiatrie und Psychotherapie, 11(3), 5-21.

Bremner JD, Vythilingam M, Vermetten E, Southwick SM, McGlashan T, Nazeer A, Khan S, Vaccarino LV, Soufer R, Garg PK, Ng CK, Staib LH, Duncan JS, Charney DS (2003): MRI and PET study of deficits in hippocampal structure and function in women with childhood sexual abuse and posttraumatic stress disorder. Am J Psychiatry 160: 924-32.

Brower, M. C. \& Price, B. H. (2001). Neuropsychiatry of frontal lobe dysfunction in violent and criminal behaviour: a critical review. Journal of Neurology, Neurosurgery \& Psychiatry, 71(6), 720-726.

Bufkin, J., L. \& Luttrell, V. R. (2005). Neuroimaging studies of aggressive and violent behavior. Current findings and implications for criminology and criminal justice. Trauma Violence \& Abuse, 6(2), 176-191.

Burgess, K. B., Marshall, P. J., Rubin, K. H. \& Fox, N. A. (2003). Infant attachment and temperament as predictors of subsequent externalizing problems and cardiac physiology. Journal of Child Psychology and Psychiatry, 44(6), 819-831.

Carlo, G., Raffaelli, M., Laible, D. J. \& Meyer, K. A. (1999). Why are girls less physically aggressive than boys? Personality and parenting mediators of physical aggression. Sex Roles, 40, 711-729.

Caspi, A., McClay, J., Moffitt, T. E., Mill, J., Martin, J. Craig, I. W.et al. (2002). Role of genotype in the cycle of violence in maltreated children. Science, 297, 851854.

Cicchetti, D. (2002). How a child builds a bain: insights from normality and psychopathology. In W. W. Hartup \& R. A. Weinberg (Hrsg.), Child Psychology in Retrospect and Prospect: In Celebration of the 75th Anniversary of the Institute of Child Development; The Minnesota Symposia on Child Psychology (Bd. 32, S. 23-71). London: LEA.

Clarke, R. A., Murphy, D. L. \& Constantino, J. N. (1999). Serotonin and externalizing behavior in young children. Psychiatry Research, 86(1), 29-40.

Davidson, R. J., Putnam, K. M. \& Larson, C. L. (2000). Dysfunction in the neural circuitry of emotion regulation-A possible prelude to violence. Science, 289 591-594.

Dodge, K. A., Lansford, J. E., Burks, V. S., Bates, J. E., Pettit, G. S., Fontaine, R.et al. (2003). Peer rejection and social information-processing factors in the development of aggressive behavior problems in children. Child Development, 74(2), 374-393.

Eisenberg, N. \& Morris, A. S. (2002). Children's emotionrelated regulation. Advances In Child Development And Behavior, 30(30), 189-229.

Hennig, J., Reuter, M., Netter, P., Burk, C. \& Landt, O. (2005). Two types of aggression are differently related 
to serotonergic activity and the A779C TPH polymorphism. Behavioral Neuroscience, 119(1), 16-25.

Huang, Y. Y., Cate, S. P., Battistuzzi, C., Oquendo, M. A., Brent, D. \& Mann, J. J. (2004). An association between a functional polymorphism in the monoamine oxidase A gene promoter, impulsive traits and early abuse experiences. Neuropsychopharmacology, 29(8), 1498-1505.

Ishikawa, S. S., Raine, A., Lencz, T., Bihrle, S. \& LaCasse, L. (2001). Autonomic stress reactivity and executive functions in successful and unsuccessful criminal psychopaths from the community. Journal of Abnormal Psychology, 110, 423-432.

Lahey, B. B., McBurnett, K. \& Loeber, R. (2000). Are attention-deficit/hyperactivity disorder and oppositional defiant disorder developmental precursors to conduct disorder? In A. Sameroff, M. Lewis \& S. M. Miller (Hrsg.), Handbook of Developmental Psychopathology (2nd ed., S. 431-446). New York: Plenum.

Lee, R. \& Coccaro, E. (2001). The neuropsychopharmacology of criminality and aggression. Canadian Journal of Psychiatry, 46(1), 35-44.

Lesch, K. P. \& Merschdorf, U. (2000). Impulsivity, aggression, and serotonin: a molecular psychobiological perspective. Behavioral Sciences and the Law, 18, 581-604.

Loeber, R., Lacourse, E. \& Homish, D. L. (2005). Homicide, violence, and developmental trajectories. In R. E. Tremblay, W. W. Hartrup \& J. Archer (Hrsg.), Developmental Origins Of Aggression (S. 202-219). New York, London: Guilford Press.

Lück, M., Strüber, D. \& Roth, G. (2005). Psychobiologische Grundlagen aggressiven und gewalttätigen Verhaltens (Bd. 5). Oldenburg: BIS.
Manuck, S. B., Flory, J. D., Ferrell, R. E., Dent, K. M., Mann, J. J. \& Muldoon, M. F. (1999). Aggression and anger-related traits associated with a polymorphism of the tryptophan hydroxylase gene. Biological Psychiatry, 45(5), 603-614.

Maras, A., Laucht, M., Fischer, T., Wilheim, C. \& Schmidt, M. H. (2006). Erniedrigte Serotoninkonzentrationen im thrombozytenfreien Plasma bei Jugendlichen mit externalen Verhaltensproblemen. Zeitschrift fur Kinder- und Jugendpsychiatrie, 34(1), 29-35.

Maras, A., Laucht, M., Gerdes, D., Wilhelm, C., Lewicka, S., Haack, D.et al. (2003). Association of testosterone and dihydrotestosterone with externalizing behavior in adolescent boys and girls. Psychoneuroendocrinology, 28(7), 932-940.

Moffitt, T. E. \& Caspi, A. (2001). Childhood predictors differentiate life-course persistent and adolescence-limited antisocial pathways among males and females. Development and Psychopathology, 13(2), 355-375.

Moffitt, T. E., Caspi, A., Harrington, H. \& Milne, B. J. (2002). Males on the life-course-persistent and adolescence-limited antisocial pathways: Follow-up at age 26 years. Development and Psychopathology, 14(1), $179-207$

New, A. S., Gelernter, J., Yovell, Y., Trestman, R. L., Nielsen, D. A., Silverman, J.et al. (1998). Tryptophan hydroxylase genotype is associated with impulsiveaggression measures: a preliminary study. American Journal of Medical Genetics, 81(1), 13-17.

Parsey, R. V., Oquendo, M. A., Simpson, N. R., Ogden, R. T., Van Heertum, R., Arango, V.et al. (2002). Effects of sex, age, and aggressive traits in man on brain serotonin 5-HT1A receptor binding potential measured by PET using [C-11]WAY-100635. Brain Research, $954,173-182$
Raine, A., Buchsbaum, M. \& Lacasse, L. (1997). Brain abnormalities in murderers indicated by positron emission tomography. Biological Psychiatry, 42(6), 495-508.

Raine, A., Ishikawa, S. S., Arce, E., Lencz, T., Knuth, K. H., Bihrle, S.et al. (2004). Hippocampal structural asymmetry in unsuccessful psychopaths. Biological Psychiatry, 55, 185-191.

Raine, A., Lencz, T., Bihrle, S., LaCasse, L. \& Colletti, P. (2000). Reduced prefrontal gray matter volume and reduced autonomic activity in antisocial personality disorder. Archives of General Psychiatry, 57(2), 119-127.

Roth, G. (2003). Fühlen, Denken, Handeln. Frankfurt am Main: Suhrkamp.

Roth, G. \& Dicke, U. (2005). Evolution of the brain and intelligence. Trends in Cognitive Sciences, 9(5), 250-257.

Spangler, G., Grossmann, K. E. \& Schieche, M. (2002). Psychobiologische Grundlagen der Organisation des Bindungsverhaltenssystems im Kleinkindalter. Psychologie in Erziehung und Unterricht, 49(2), 102-120.

Statistisches Bundesamt. (2006). Rechtspflege. Strafverfolgung - Lange Reihen über verurteilte Deutsche und Ausländer nach Art der Straftat, Altersklassen und Geschlecht. Wiesbaden.

van Goozen, S. H. M., Matthys, W., Cohen-Kettenis, P. T., Westenberg, H. \& van Engeland, H. (1999). Plasma monoamine metabolites and aggression: two studies of normal and oppositional defiant disorder children. European Neuropsychopharmacology, 9(1-2), 141-147.

Yang, Y., Raine, A., Lencz, T., Bihrle, S., LaCasse, L. \& Colletti, P. (2005). Volume reduction in prefrontal gray matter in unsuccessful criminal psychopaths. Biological Psychiatry, 57, 1103-1108.

\section{Psychische Störungen bei Straftätern}

Günter Hinrichs und Denis Köhler

\section{Forensisch relevante Krankheitsbilder}

»Krank und / kriminell « ist ein Stichwort, das man häufig antrifft, wenn es um interdisziplinäre Forschung geht, etwa auf der 18. (forensischen) Eickelborner Fachtagung im Jahre 2003, zurückgehend auf ein Gutachten von Rasch aus dem Jahre 1983, welches die Grundlage für die Entwicklung einer eigenständigen forensisch-psychiatrischen Klinik in Lippstadt-Eickelborn bildete. Drei Jahre später äußerte sich Rasch (1986) eingehend zu forensisch relevanten psychologisch-psychiatrischen Störungsbildern und formulierte seinen strukturellsozialen Krankheitsbegriff. Danach soll für einen Straftäter beurteilt werden, ob ein bestimmter $\mathrm{Zu}$ stand bei ihm die Struktur von »Krankheit« habe und ob er die allgemeine soziale Kompetenz (Dimensionen: Einengung der Lebensführung, Arbeitsunfähigkeit, Abbruch bzw. Verlust von Kontakten, verzerrte Realitätsbeurteilung, Stereotypisierung des Verhaltens, festgelegt sein auf bestimmte Verhaltensmuster, Häufung sozialer Konflikte auch außerhalb strafrechtlicher Belange) der Persönlichkeit beeinträchtige (Rasch 1986, S. 43, 44). Einfach gesprochen werden damit psychische und soziale Komponenten für Kriminalität in einem Stufenmodell verbunden, wobei psychologisch- psychiatrische Handicaps quasi die Schädigungsgrundlage bilden, aus der sich ein Sozialversagen mit Affinität zu strafbarem Verhalten ergibt. Auch wenn dieser Krankheitsbegriff unter wissenschaftlichen Kriterien deutliche Schwächen aufweist, gelingt ihm in zusammenfassender Betrachtung die Berücksichtigung psychischer und sozialer Phänomene in Bezug auf kriminelles Verhalten.

\section{Historische Entwicklung}

Betrachtet man die geschichtliche Entwicklung, so ist die Berücksichtigung psychischer Phänomene in Verbindung mit Kriminalität relativ neu. Zwar wurden gerade bei ausgefallenen Straftaten immer wieder seelische Begleitumstände in Einzelfällen herausgestellt, eine systematische Betrachtung setzt jedoch erst zu Beginn des letzten Jahrhunderts mit der Psychoanalyse ein. Freud (1916) beschrieb den sog. neurotischen Verbrecher aus Schuldbewusstsein noch unter recht mechanistischen Vorstellungen, schon mit Aichhorns (1926) Arbeiten ist eine Erweiterung um pädagogische Aspekte verbunden. Unter dem Bemühen um eine Differenzierung des Verwahrlosungs- bzw. Dissozialitätsbegriffes entstand die Vorstellung der neurotischen
Delinquenz prototypisch etwa als wiederholter Kaufhausdiebstahl von Dingen, die weder dem eigenen Nutzen noch dem materiellen Gewinn dienen, rasch entdeckt werden, ggf. unter demonstrativem Verhalten, die seelische Erschütterung scheint dann oberflächlich zu kontrastieren mit dem Wiederholungszwang. Aber auch schwerwiegende Delikte wie aggressive Beziehungs- und Konflikttaten sowie einige Sexualstraftaten werden mit diesem Begriff gekennzeichnet, der sozusagen die spezifische Ätiologie einer strafbaren Handlung unter besonderer Berücksichtigung psychischer Konflikte aufzeigt. Neuere psychoanalytische Vorstellungen beschäftigen sich unter psychodynamischen Gesichtspunkten auch mit der chronischdissozialen Entwicklung, womit eine Verbindung zu den Persönlichkeitsstörungen hergestellt wird.

\section{Der medizinisch-psychiatrische Krankheits- begriff der forensischen Psychiatrie}

Die Beschäftigung der forensischen Psychiatrie mit Straftätern und kriminellem Verhalten bestand lange Zeit in entsprechender gutachterlicher Tätigkeit unter besonderer Fragestellung und der Verwahrung der psychiatrisch erkrankten Rechtsbre- 